\title{
ON THE CONVERGENCE OF A SOLUTION OF A DIFFERENCE EQUATION TO A SOLUTION OF THE EQUATION OF DIFFUSION ${ }^{1}$
}

\section{L. JUNCOSA AND DAVID YOUNG}

1. Introduction. Let $f(x)$ be a Lebesgue integrable function satisfying the $l_{c}$ condition (see, e.g., Hardy $[3$, p. 359])

$$
\int_{0}^{t}[f(x+u)+f(x-u)-2 c(x)] d u=o(t)
$$

for each $x$ in $0 \leqq x \leqq 1$. If $a_{n}=2 \int_{0}^{1} f(x) \sin n \pi x d x, n=1,2, \cdots$, then

$$
u(x, t)=\sum_{n=1}^{\infty} a_{n} \sin n \pi x e^{-n^{2} \pi^{2} t}
$$

is the Fourier series solution in $R: 0<x<1, t>0$, of the partial differential equation of diffusion

$$
\frac{\partial u}{\partial t}=\frac{\partial^{2} u}{\partial x^{2}}
$$

Furthermore, (1) satisfies the boundary conditions $u(+0, t)$ $=u(1-0, t)=0, t>0$ and as a consequence of $\S 1$ and $\S 3$ of Appendix II of [3] it also satisfies the initial condition $u(x,+0)=f(x)$ at every point of continuity of $f(x)$ in $0<x<1$ as well as the condition $u(x,+0)$ $=(1 / 2)[f(x+0)+f(x-0)]$ at every point $x$ where $f(x)$ possesses these one-sided limits. Moreover, from a slight modification of Hardy and Rogosinski [4, p. 66] on Abel summability of Fourier series and from Theorems 270 (due to M. L. Cartwright [1]) and 273 in Appendix $\mathrm{V}$ of [3] it follows that as $t \rightarrow 0+$,

$$
\lim u(x, t)=f(x)
$$

uniformly in any closed interval of continuity of $f(x)$. The uniformity of the limit goes back to the original theorems of Fejér [2].

Now for the remainder of this note let $f(x)$ be continuous in $0 \leqq x \leqq 1$ except for at most $N$ points at which it may have a finite jump. (Then all the preceding remarks are still applicable.) Let $M$ be a positive integer variable, $\Delta x=M^{-1}$, and $\Delta t=r(\Delta x)^{2}$. Then if

Presented to the Society, April 25, 1953; received by the editors June 16, 1952 and, in revised form, April 20, 1953 and June 25, 1953.

1 This paper was prepared in part by research at the Ballistic Research Laboratories under Project No. TB3-0007 K. 


$$
b_{n}=b_{n}(M)=(2 / M) \sum_{j=1}^{M-1} f(j / M) \sin (n \pi j / M), \quad n=1,2, \cdots, M-1,
$$

an analogue of the Fourier series (1)

$$
U_{M}(x, t)=\sum_{n=1}^{M-1} b_{n} \sin n \pi x\left[1-4 r \sin ^{2}(n \pi / 2 M)\right]^{t M^{2} / r}
$$

is an exact solution (see, e.g., [7]) of a partial difference equation analogue of (2)

$$
\begin{aligned}
U_{M}(x, t+\Delta t) & -U_{M}(x, t) \\
& =r\left[U_{M}(x+\Delta x, t)+U_{M}(x-\Delta x, t)-2 U_{M}(x, t)\right]
\end{aligned}
$$

for $(x, t),(x+\Delta x, t),(x-\Delta x, t)$, and $(x, t+\Delta t)$ in $R$. It also satisfies exactly the boundary conditions $U_{M}(0, t)=U_{M}(1, t)=0, t \geqq 0$ and the initial condition $U_{M}(x, 0)=f(x)$ for those $x$ in $0<x<1$ for which $M x$ is an integer.

When it converges, the series

$$
V_{M}(x, t)=\sum_{n=1}^{\infty} c_{n} \sin n \pi x\left[1-4 r \sin ^{2}(n \pi / 2 M)\right]^{t M^{2} / r},
$$

where $c_{n}=c_{n}(M), n=1,2, \cdots$, also satisfies (4) but not, in general, the corresponding boundary conditions except when $c_{n}=b_{n}$ for $n \leqq M-1$ and $c_{n}=0$ for $n \geqq M$.

Recent interest in the problem of convergence as $M \rightarrow \infty$ has resulted in a proof by Leutert [6] of the convergence of (5) to (1) for the case where $0<r \leqq 1 / 4$ and $\left|c_{n}-a_{n}\right| \rightarrow 0$ uniformly for $n<M$ and for $f(x)$ sectionally continuous with one-sided derivatives everywhere. Hildebrand [5] proved convergence of (3) to (1) for $0<r \leqq 1 / 2$ when $f(x)$ has bounded variation and is continuous except for a finite number of finite jumps. He had more severe restrictions on $f(x)$ when $r=1 / 2$. With our considerably more general $f(x)$ we shall prove convergence of (3) to (1) for $0<r \leqq 1 / 2$ and convergence of (5) to (1) for all $r>0$.

2. Two lemmas. Let $\beta$ be an arbitrary, fixed number in $0<\beta<1$ and define

$$
k=k(M, r, \beta)=\left\{\begin{array}{lr}
\langle M / 3\rangle, & 0<r \leqq 1 / 2, \\
\left\langle(2 M / \pi) \sin ^{-1}(\beta / 4 r)^{1 / 2}\right\rangle, & r>1 / 2,
\end{array}\right.
$$

where $\langle x\rangle$ represents the greatest integer not exceeding $x$.

LEMMA 1. Uniformly for all positive integers $M$ and all $t \geqq t_{0}>0$, 
we have, for $0<r \leqq 1 / 2$,

$$
\sum_{n=1}^{M-1}\left|1-4 r \sin ^{2}(n \pi / 2 M)\right|^{t \cdot M^{2} / r}=O(1)
$$

and, for $r>0$,

$$
\sum_{n=1}^{k}\left|1-4 r \sin ^{2}(n \pi / 2 M)\right|^{t M^{2} r}=O(1)
$$

where $k$ is defined by (6).

Proof. Let $0<r \leqq 1 / 2$. Then, for $n \leqq\langle M / 2\rangle$,

$-1+4 r \sin ^{2}(n \pi / 2 M)$

$$
\begin{aligned}
& \leqq-1+4 r \sin ^{2} \frac{M-n}{2 M} \pi=-1+4 r \cos ^{2}(n \pi / 2 M) \\
& =4 r-1-4 r \sin ^{2}(n \pi / 2 M) \leqq 1-4 r \sin ^{2}(n \pi / 2 M) .
\end{aligned}
$$

Using (9) and the inequalities $\log (1-z) \leqq-z$ for $0 \leqq z<1$ and $2 z / \pi \leqq \sin z$ for $0 \leqq z \leqq \pi / 2$, we have

$$
\begin{aligned}
\sum_{n=1}^{M-1}\left|1-4 r \sin ^{2}(n \pi / 2 M)\right| & \mid M^{2} / r \\
& \leqq 2 \sum_{n=1}^{\langle M / 2\rangle}\left[1-4 r \sin ^{2}(n \pi / 2 M)\right]^{t M^{2} / r} \\
& \leqq 2 \sum_{n=1}^{\langle M / 2\rangle} e^{-4 M^{2} t \sin ^{2}(n \pi / 2 \cdot M)}<2 \sum_{n=1}^{\langle M / 2\rangle} e^{-4 n^{2} t}=O(1) .
\end{aligned}
$$

For the larger range of $r, r>0,(8)$ is proved using the same inequalities in the same manner.

LEMMA 2. For $n \leqq k, r>0$, and $t \geqq t_{0}>0$,

(10) $\delta \equiv\left|\left[1-4 r \sin ^{2}(n \pi / 2 M)\right]^{t M^{2} / r}-e^{-n^{2} \pi^{2} t}\right| \leqq C t\left(n^{4} \pi^{4} / 4 M^{2}\right) e^{-n^{2} \alpha^{2} \pi^{2} t}$ where $C=\max \{1 / 3,2 r /(1-\beta)\}$ and

$$
\alpha^{2}=1-\max \left\{\pi^{2} / 108,(1 / 3)\left[\sin ^{-1} \beta / 4 r\right]^{2}\right\} .
$$

Proof. By the Mean Value Theorem we have, for $n \leqq k$,

$$
\delta=n^{2} \pi^{2} t\left|\left(M^{2} / n^{2} \pi^{2} r\right) \log \left[1-4 r \sin ^{2}(n \pi / 2 M)\right]+1\right| e^{-\xi \pi^{2} t}
$$

for some $\xi$ lying between $n^{2}$ and $-\left(M^{2} / \pi^{2} r\right) \log \left[1-4 r \sin ^{2}(n \pi / 2 M)\right]$. From the elementary inequalities, $-z-z^{2} / 2(1-z) \leqq \log (1-z) \leqq-z$ for $0 \leqq z<1, \sin z \leqq z$ for $z \geqq 0$, and $\sin ^{2} z \geqq z^{2}-z^{4} / 3$ for $|z|<\pi / 2$, 


$$
\begin{aligned}
\frac{-2 r}{1-\beta}\left(\frac{n \pi}{2 M}\right)^{2} & \leqq \frac{-2 r}{1-4 r \sin ^{2}(n \pi / 2 M)}\left(\frac{n \pi}{2 M}\right)^{2} \\
& =-\frac{1}{r}\left(\frac{M}{n \pi}\right)^{2}\left[4 r\left(\frac{n \pi}{2 M}\right)^{2}+\frac{8 r^{2}(n \pi / 2 M)^{4}}{1-4 r \sin ^{2}(n \pi / 2 M)}\right]+1 \\
& \leqq\left(\frac{M}{n \pi}\right)^{2} \frac{1}{r} \log \left[1-4 r \sin ^{2}(n \pi / 2 M)\right]+1 \\
& \leqq-4(M / n \pi)^{2} \sin ^{2}(n \pi / 2 M)+1 \\
& \leqq-(2 M / n \pi)^{2}\left[(n \pi / 2 M)^{2}-(1 / 3)(n \pi / 2 M)^{4}\right]+1 \\
& =(1 / 3)(n \pi / 2 M)^{2} .
\end{aligned}
$$

The last inequality of (11) yields

$$
\xi \geqq n^{2}\left[1-(1 / 3)(n \pi / 2 M)^{2}\right] .
$$

From (11) and (12) we obtain (10).

\section{3 . Convergence theorems.}

THEOREM 1. For any fixed $t_{0}>0$ and for $0<r \leqq 1 / 2$,

$$
\lim _{M \rightarrow \infty} U_{M}(x, t)=u(x, t)
$$

uniformly for $0 \leqq x \leqq 1$ and $t \geqq t_{0}$.

Proof. For $k=1,2, \cdots$, let

$$
\sigma_{k}(x, t)=\sum_{n=1}^{k} A_{k, n} \sin n \pi x e^{-n^{2} \pi^{2} t}
$$

be the arithmetic mean of the first $k$ partial sums of (1). Then

$$
A_{k, n}=\left\{\begin{array}{lr}
(k-n+1) a_{n} / k, & 1 \leqq n \leqq k, \\
0, & n>k .
\end{array}\right.
$$

For $k$ as defined in (6) we have

$$
\left|U_{M}(x, t)-u(x, t)\right| \leqq\left|E_{1}(x, t)\right|+\left|E_{2}(x, t)\right|+\left|E_{3}(x, t)\right|
$$

where

$$
\begin{aligned}
& E_{1}(x, t)= \sum_{n=1}^{M-1}\left(b_{n}-A_{k, n}\right) \sin n \pi x\left[1-4 r \sin ^{2}(n \pi / 2 M)\right] t M^{2} / r \\
& E_{2}(x, t)=\sum_{n=1}^{k} A_{k, n} \sin n \pi x\left\{\left[1-4 r \sin ^{2}(n \pi / 2 M)\right] t M^{2} / r\right. \\
&\left.-e^{-n^{2} \pi^{2} t}\right\}
\end{aligned}
$$


and

$$
E_{3}(x, t)=\sum_{n=1}^{k} A_{k, n} \sin n \pi x e^{-n^{2} \pi^{2} t}-u(x, t) .
$$

Let us cover the set of points of discontinuity of $f(x)$ by a set $E$ which is the sum of a finite number of open intervals, the sum, $\eta$, of whose lengths is arbitrarily small. Let $I$ be the interval $0 \leqq x \leqq 1$. Then, on $I-I E$, by the corollary to Fejér's principal theorem on summability $(C, 1)$ of Fourier series (see Fejér $\left[2\right.$, p. 60]), $\sigma_{k}(x, 0)$ converges uniformly to $f(x)$. On $I E,\left|\sigma_{k}(x, 0)-f(x)\right| \leqq 2 F$ where $F=$ LUB $|f(x)|$ in $0 \leqq x \leqq 1$. Then, for all sufficiently large $M$ we have

$$
\begin{aligned}
\left|b_{n}-A_{k, n}\right| & =\frac{2}{M}\left|\sum_{j=1}^{M-1}\left[f(j / M)-\sigma_{k}(j / M, 0)\right] \sin (n \pi j / M)\right| \\
& <(2 / M)[M \cdot o(1)+2 F(\eta M+N)]=o(1)
\end{aligned}
$$

uniformly for all $n \leqq M$. Hence, from (16) and (7) of Lemma 1, we have that the first member of the right-hand side of (13) is $o(1)$ uniformly for $0 \leqq x \leqq 1$ and $t \geqq t_{0}$.

Using $\left|A_{k, n}\right| \leqq\left|a_{n}\right| \leqq 4 F / \pi$ and Lemma 2 , we have

$$
\left|E_{2}(x, t)\right| \leqq\left(F C t \pi^{3} / M^{2}\right) \sum_{n=1}^{k} n^{4} e^{-n^{2} \alpha^{2} \pi^{2} t}=O\left(M^{-2}\right)
$$

uniformly in $0 \leqq x \leqq 1$ and $t \geqq t_{0}$.

Finally, since for sufficiently large $k$

$$
\begin{aligned}
\left|A_{k, n}-a_{n}\right| & \leqq 2 \int_{0}^{1}\left|\sigma_{k}(y, 0)-f(y)\right||\sin n \pi y| d y \\
& \leqq(4 / \pi)\left[o(1)+2 F_{\eta}\right]=o(1)
\end{aligned}
$$

we have

$$
\left|E_{3}(x, t)\right| \leqq o(1) \cdot \sum_{n=1}^{\infty} e^{-n^{2} \pi^{2} t}=o(1)
$$

uniformly for $0 \leqq x \leqq 1$ and $t \geqq t_{0}$, completing the proof of the theorem.

Theorem 2. Let $c_{n}=c_{n}(M)$ in (5) be such that $c_{n}=0$ for $n>k$ (where $k$ is defined by (6)) and

$$
\lim _{M \rightarrow \infty}\left|c_{n}-a_{n}\right|=0
$$

uniformly for $n \leqq k$. Then, for each $r>0$, 


$$
\lim _{M \rightarrow \infty} V_{M}(x, t)=u(x, t)
$$

uniformly in $0 \leqq x \leqq 1$ and $t \geqq t_{0}>0$.

Proof: We have

$$
\left|V_{M}(x, t)-u(x, t)\right| \leqq\left|E_{1}^{*}(x, t)\right|+\left|E_{2}(x, t)\right|+\left|E_{3}(x, t)\right|
$$

where $E_{2}(x, t)$ and $E_{3}(x, t)$ are defined by (14) and (15) and

$$
E_{1}^{*}(x, t)=\sum_{n=1}^{k}\left(c_{n}-A_{k, n}\right) \sin n \pi x\left[1-4 r \sin ^{2}(n \pi / 2 M)\right]^{t M^{2} / r} .
$$

Using (17) and (18) in the triangle inequality, we get $c_{n}-A_{k, n}=o(1)$. Using this and (8) of Lemma 1 , we obtain $E_{1}^{*}(x, t)=o(1)$ uniformly for $0 \leqq x \leqq 1$ and $t \geqq t_{0}>0$. From the proof of Theorem 1 , we have $E_{2}(x, t)+E_{3}(x, t)=o(1)$ uniformly for $0 \leqq x \leqq 1$ and $t \geqq t_{0}$, thus completing the proof of the theorem.

In the case $0<r \leqq 1 / 2, U_{M}(x, 0)$ satisfies the initial conditions on a set asymptotically dense on $0 \leqq x \leqq 1$ as $M \rightarrow \infty$. On the other hand, (18) is not sufficient for $V_{M}(x, 0+)$ to satisfy the initial conditions or even be bounded. This is easily seen in the case where $c_{n}(M)=a_{n}$ $+(1 / M) \sin (n \pi / 2)$ for $n \leqq M-1$ and $c_{n}(M)=0$ for $n \geqq M$. However, $c_{n}=a_{n}+o(1 / M)$ is clearly sufficient for convergence for $t=0$.

Theorem 1 assures uniform convergence of $U_{M}(x, t)$ to $u(x, t)$ for $t \geqq t_{0}$ and $0 \leqq x \leqq 1$. However, $u(x, t)$ is real for every $(x, t)$ in $R$ and on its boundaries, while for $1 / 4<r \leqq 1 / 2$ and those $(x, t)$ such that $t M^{2} / r$ is not an integer, $U_{M}(x, t)$ may be complex. Therefore, we shall state two more theorems covering a general class of real interpolations on $U_{M}(x, t)$ and $V_{M}(x, t)$, which include, e.g., bilinear interpolation. Let $P_{1}, P_{2}, P_{3}$, and $P_{4}$ be the points at the corners of an elemental rectangle of area $\Delta x \Delta t$. Then, if $\alpha_{i}(x, t), i=1, \cdots, 4$, are non-negative functions whose sum is unity and if $\bar{W}_{M}(x ; t)$ $=\sum_{i=1}^{4} \alpha_{i}(x, t) W_{M}\left(P_{i}\right)$, then we say $\bar{W}_{M}(x, t)$ is a four-point interpolation on $W_{M}(x, t)$ satisfying a "maximum-minimum principle." In our definition we also assume that, for $(x, t)$ on the boundary of an elementary rectangle, $\bar{W}_{M}(x, t)$ is determined solely by interpolation on the two neighboring meshpoints determining the straightline segment of the boundary containing $(x, t)$.

TheOREM 3. If $\bar{U}_{M}(x, t)$ is a four-point interpolation on $U_{M}(x, t)$ satisfying a "maximum-minimum principle," then

$$
\lim _{M \rightarrow \infty} \bar{U}_{M}(x, t)=u(x, t)
$$


uniformly in $0 \leqq x \leqq 1$ and $t \geqq t_{0}>0$. Furthermore, uniformly on $I-I E$

$$
\lim _{M \rightarrow \infty} \bar{U}_{M}(x, 0)=f(x) \text {. }
$$

Proof. For any $P_{1}, P_{2}, P_{3}$, and $P_{4}$ as defined above we have

$$
\begin{aligned}
\left|\bar{U}_{M}(x, t)-u(x, t)\right| \leqq & \sum_{i=1}^{4} \alpha_{i}\left|U_{M}\left(P_{i}\right)-u\left(P_{i}\right)\right| \\
& +\sum_{i=1}^{4} \alpha_{i}\left|u\left(P_{i}\right)-u(x, t)\right| .
\end{aligned}
$$

Then, (19) follows immediately from Theorem 1 and the uniform continuity of $u(x, t)$ for $t \geqq t_{0}$. Similarly, if $\left(x_{1}, 0\right)$ and $\left(x_{2}, 0\right)$ are adjacent meshpoints such that $x_{1} \leqq x \leqq x_{2}$ is in $I-I E$, then, since $\bar{U}_{M}\left(x_{i}, 0\right)$ $=f\left(x_{i}\right)$ and $f(x)$ is uniformly continuous on $I-I E$, we have

$$
\left|\bar{U}_{M}(x, 0)-f(x)\right| \leqq \sum_{i=1}^{2} \alpha_{i}\left|f\left(x_{i}\right)-f(x)\right|=o(1) .
$$

Similarly, one can prove the following theorem.

THEOREM 4. If $\bar{V}_{M}(x, t)$ is a four point interpolation on $V_{M}(x, t)$ satisfying a "maximum-minimum" principle and if (18) holds, then

$$
\lim _{M \rightarrow \infty} \bar{V}_{M}(x, t)=u(x, t)
$$

uniformly in $0 \leqq x \leqq 1$ and $t \geqq t_{0}>0$.

\section{REFERENCES}

1. M. L. Cartwright, On the relation between different types of Abel summation, Proc. London Math. Soc. (2) vol. 31 (1930) pp. 81-96. $51-69$

2. L. Fejér, Untersuchungen über Fouriersche Reihen, Math. Ann. vol. 58 (1904) pp.

3. G. H. Hardy, Divergent series, Oxford, 1949. 1944.

4. G. H. Hardy and W. IV. Rogosinski, Fourier series, Cambridge Tract No. 38,

5. F. B. Hildebrand, On the convergence of numerical solutions of the heat-flow equation, Journal of Mathematics and Physics vol. 31 (1952) pp. 35-41.

6. W. W. Leutert, On the convergence of unstable approximate solutions of the heat equation to the cxact solution, Journal of Mathematics and Physics vol. 30 (1952) pp. 245-251.

7. G. G. O'Brien, M. A. Hyman, and S. Kaplan, A study of the numerical solution of partial differential equations, Journal of Mathematics and Physics vol. 29 (1951) pp. 223-251.

RAND CORPORATION AND

UNIVERSITY OF MARYLAND 